\title{
Análise sobre a Contribuição da Tecnologia Blockchain na Gestão da Cadeia de Supri- mentos
}

\section{Analysis on the Contribution of Blockchain Technology in Supply Chain Management}

\author{
Daniela Maria Feltrin Marchini \\ Faculdade de Tecnologia de Americana - FATEC - Brasil \\ daniela.marchini@fatec.sp.gov.br \\ ORCID: 0000-0003-1282-6574 \\ João Batista de Camargo Junior \\ Universidade Metodista de Piracicaba - UNIMEP - Brasil \\ joao.junior2@unimep.br \\ ORCID: 0000-0002-2174-7221 \\ Silvio Roberto Ignacio Pires \\ Fundação Getulio Vargas - FGV - Brasil \\ sripires@fgvmail.com \\ ORCID: 0000-0002-3686-2142
}

Submetido em 08/04/2020; Aprovado em 06/06/2020.

\begin{abstract}
Resumo
Objetivos do estudo: 0 objetivo da pesquisa é identificar como o Blockchain pode contribuir com a Gestão da Cadeia de Suprimentos. Metodologia/abordagem: Foi realizada pesquisa bibliográfica com a aplicação de revisão sistemática da literatura na base de dados da CAPES, utilizando como chave de busca as palavras Supply Chain Management e Blockchain no título e no assunto, seguida de entrevistas com profissionais especialistas na área para a validação e complementação das características levantadas. Principais resultados: Inicialmente, foram identificados onze artigos que demonstraram diferentes formas de contribuição do Blockchain para a utilização em Gestão da Cadeia de Suprimentos, tendo como características mais relevantes o registro distribuído, a imutabilidade e o custo. Os especialistas adicionaram a eliminação de entidades de verificação, de disputas entre elos e redução de perdas. Contribuições acadêmicas: 0 trabalho apresenta exemplos de diferentes aplicações do Blockchain na Gestão da Cadeia de Suprimentos discutidas nos artigos selecionados, podendo servir de base para estudos para novas utilizações em diferentes áreas e setores. Contribuições práticas: 0 estudo demonstrou que o Blockchain pode contribuir de diferentes formas para as operações na Gestão da Cadeia de Suprimentos e, também, identificou barreiras a serem superadas para sua ampla operacionalização, como a necessidade de recursos computacionais robustos e regulamentações.
\end{abstract}

Palavras-chave: Blockchain, Gestão da Cadeia de Suprimentos, Tecnologia de Informação

\section{Abstract}

Study purpose: The objective of this research is to identify how Blockchain can contribute to Supply Chain Management. Methodology / approach: Bibliographic research was carried out with the application of systematic literature review in CAPES database, using the words Supply Chain Management and Blockchain in the title and subject as the search key, followed by interviews with professionals in the field for validation and complementation of the characteristics raised. Main findings: Initially, eleven articles were identified that demonstrated different forms of Blockchain contribution for use in Supply Chain Management, having distributed registration, immutability and cost as the most relevant characteristics. Experts added the elimination of verification entities, link disputes and loss reduction. Academic contributions: The study presents examples of different Blockchain applications in Supply Chain Management discussed in selected articles and can serve as a basis for studies for new use in different areas and sectors. Practical contributions: The study demonstrated that Blockchain can contribute in different ways to operations in Supply Chain Management and also identified barriers to be 
overcome for its wide operationalization, such as the need for robust computing resources and regulations.

Keywords: Blockchain, Supply Chain Management, Information Technology

\section{Introdução}

Os avanços tecnológicos ocorridos nas últimas décadas têm transformado a forma como as organizações produzem e gerenciam seus negócios. Entre as diversas tecnologias que vêm transformando a relação entre as organizações está o Blockchain (Schwab, 2018). Para Queiroz e Wamba (2019), o Blockchain se trata de um banco de dados distribuído, executado em um ambiente compartilhado e sincronizado, que demanda que as informações sejam validadas pelos usuários. Para tanto, os dados são organizados em blocos imutáveis, e a cada novo dado a ser inserido é gerado um novo bloco com os dados do bloco anterior.

O uso desta tecnologia é conhecido na área financeira por conta do Bitcoin, mas segundo Kshetri (2018), o Blockchain tem sido experimentado em outras áreas, como na Gestão da Cadeia de Suprimentos (Supply Chain Management - SCM) . Todavia, considerando a contemporaneidade do tema, ainda não estão totalmente claros para profissionais da área gerencial quais são os principais benefícios que o Blockchain pode trazer para a gestão conjunta das atividades empresariais e como essa tecnologia poderia ser utilizada em diferentes organizações.

Nesse contexto, o objetivo principal deste artigo é identificar como o Blockchain pode contribuir com a Gestão da Cadeia de Suprimentos.

Para atingir esse objetivo, se fez necessário desenvolver como objetivos específicos a construção de um referencial teórico sobre Gestão da Cadeia de Suprimentos e sobre Blockchain, analisar o que já foi publicado sobre o uso de Blockchain em SCM com a realização de uma revisão sistemática e entrevistas com especialistas para verificar as evidências encontradas. A pesquisa se justifica, pois ainda são poucos os artigos que tratam sobre a utilização do Blockchain em SCM, conforme evidenciado na revisão sistemática. Assim, o manuscrito busca contribuir para o campo gerencial, clarificando características e contribuições da tecnologia para gestores, e para o campo acadêmico, ao avançar no debate de um assunto ainda em desenvolvimento na área de Administração.

\section{Gestão da Cadeia de Suprimentos}

Segundo Christopher (2011), uma Cadeia de Suprimentos (CS) é um conjunto de empresas conectadas e interdependentes que trabalham em regime de cooperação para gerenciar e controlar os fluxos de materiais, financeiros e de informações que perpassam todas as organizações até chegar ao cliente final. Essas ações visam à coordenação das relações entre as empresas, objetivando resultados mais lucrativos para os integrantes das cadeias de suprimentos.

Bowersox, Closs, Cooper e Bowersox (2014) afirmam que a colaboração tem o objetivo de melhorar a eficiência operacional e o posicionamento estratégico. Desta forma, as operações da cadeia de suprimentos exigem processos gerenciais que atravessam as fronteiras organizacionais e áreas funcionais, conectando fornecedores, parceiros comerciais e clientes desde a compra inicial de material até a entrega de bens e serviços.

Nesse contexto, a Gestão da Cadeia de Suprimentos (SCM) surgiu como uma resposta das empresas à necessidade dos consumidores de maior agilidade e flexibilidade. Segundo Asgari, Nikbakhsh, Hill e Farahani (2016) e Ellram e Cooper (2014), a primeira publicação sobre o tema foi realizada por dois consultores, Oliver e Weber, em 1982.

A SCM foi estabelecida como uma nova forma de estratégia gerencial que passou a ser posteriormente estudada pelo meio acadêmico com o objetivo de aumentar a competitividade das organizações por meio da oportunidade de reduzir custos e aumentar o valor dos produtos. Desse modo, a SCM iniciou, de forma não estruturada, a busca pelo aumento da competitividade e acabou se fortalecendo de forma globalizada. Isso porque, por meio da SCM, as organizações vêm desenvolvendo novos diferenciais que vão desde novas formas de gerenciar e desenvolver negócios até inovações tecnológicas, de materiais, novas formas de relacionamento com os clientes e diferentes estratégias de marketing (Pires, 2016).

A base da SCM está no comportamento colaborativo para atingir o objetivo de atender o cliente 
de forma eficiente e com o menor custo possível (Ellram \& Cooper, 2014). Bowersox et al. (2014) acrescentam que o comportamento cooperativo pode reduzir riscos e aumentar a eficiência de todos os processos, eliminando trabalhos duplicados e improdutivos, aumentando a velocidade, confiabilidade, flexibilidade, custos e qualidade, além de outros benefícios estratégicos.

Para que o processo que interliga as relações entre as empresas ocorra de maneira efetiva, um dos pontos importantes a ser gerenciado é o fluxo de informações (Christopher, 2011). Esse fluxo tem, a cada dia, ganhado nova importância para as operações conjuntas, especialmente por conta do crescimento das aplicações de e-business e e-commerce e do uso da internet como tecnologia a serviço da SCM. Considerando que a troca de dados é essencial para que as operações da cadeia ocorram, e que esses dados precisam ser confiáveis e estar disponíveis em tempo hábil, a menor falha no fluxo de informações pode causar rupturas nos processos de negócios das empresas que compõem a cadeia (Pires, 2016).

Em uma pesquisa sobre as publicações em SCM até o ano de 2015, Asgari, Nikbakhsh, Hill e Farahani (2016) identificaram algumas áreas que poderiam ser exploradas em novas publicações. Dentre as áreas destacadas, estava o gerenciamento de risco e de rupturas. Segundo os autores, futuras pesquisas poderiam entender o impacto em fatores que possam gerar rupturas em processos ou riscos para as CS, entre eles o crescimento da área computacional e técnicas de otimização, tema que o Blockchain se encaixa.

\subsection{Blockchain}

0 crescimento do uso de tecnologias de informação e comunicação tem proporcionado às empresas a oportunidade de construir novos modelos e processos para suportar a complexidade do ambiente de negócios atual, principalmente, envolvendo as áreas de logística e SCM (Queiroz \& Wamba, 2019). Entre as tecnologias que têm se apresentado com o potencial de remodelar a SCM está o Blockchain, conforme aponta Kshetri (2018), que enfatiza o crescimento do uso desta tecnologia fora da área financeira.

O Blockchain começou a ser utilizado no mercado de criptomoedas, e sua primeira publicação foi realizada por Nakamoto, em 2008. Trata-se de uma tecnologia de registro de dados distribuída e sincronizada, de formato imutável, pela qual cada nova informação gera um novo registro em cadeia (Apte \& Petrovsky, 2016). Caracteriza-se, assim, como um banco de dados com registros distribuídos e compartilhados entre todos os membros que concordaram em fazer parte da rede.

Esta forma de trabalho da tecnologia gera uma lista crescente de registros criptografados que garante que os dados estejam protegidos contra adulteração e ocultação. Kim e Laskowski (2018) e Schwab (2018) comparam a tecnologia a um livro digital de transações que pode ser programado para registrar praticamente tudo de valor e importância para a humanidade.

Uma unidade de Blockchain, segundo Treiblmaier (2018), é uma única transação de informações que envolve uma ou mais entidades. 0 autor explica que para cada registro é gerada uma cadeia de caracteres alfanuméricos de cumprimento fixo (hash), que é utilizada para codificar o conteúdo de cada transação e recriar as transações originais. A cada nova informação é criado um novo bloco que é acrescentado à cadeia de blocos anteriores, o que caracteriza o nome Blockchain.

A estrutura da tecnologia permite a implantação de vários serviços de aplicativos e tem como base a rede distribuída com tecnologia de encadeamento, validação com técnicas de consenso e a utilização de tecnologias de segurança como hash, assinatura digital e criptografia (Yoo \& Won, 2018).

0 centro da tecnologia Blockchain é a capacidade de criar e trocar registros digitais exclusivos, de modo que toda a operação é realizada sem a necessidade de controle de uma parte confiável e centralizada. Os registros exclusivos combinados à criptografia e à utilização de redes ponto a ponto (P2P - Peer to Peer) garantem transparência e precisão das informações compartilhadas, além da manutenção de seu registro histórico (Schwab, 2018). Schwab (2018) ainda salienta que estas características criam a chamada "internet de valor" devido à transferência de materiais digitais exclusivos, livres do risco de cópias falsas ou duplo envio. Porém, as mesmas questões que garantem os aspectos positivos da tecnologia também podem se tornar pontos de preocupação, uma vez que estas redes que garantem a segurança e reduzem os custos das organizações também podem ser utilizadas para fins ilícitos e criminosos.

Segundo Treiblmaier (2018), o Blockchain não é uma tecnologia isolada, já que opera junto com outras tecnologias, o que inclui a internet, permitindo armazenar registros e transações utilizando regras de consenso e contratos inteligentes (Smart Contracts). Um contrato inteligente, segundo Yoo e 
Won (2018), é um protocolo que tem a função de substituir a necessidade de um contrato. Ele automatiza itens da negociação como confirmações e cláusulas contratuais sobre a execução, reduzindo custos transacionais relacionados ao contrato. Min (2018) complementa que um contrato inteligente tem o objetivo de facilitar, verificar e impor obrigações contratuais por meio da autoverificação e autoexecução das regras e penalidades acordadas previamente (como a troca de dinheiro, ações, entre outros), sem a necessidade de um intermediário.

Outro importante ponto a ser analisado é que existem diferentes arquiteturas de Blockchain: público (aberto) ou privado (fechado). Esta classificação está relacionada à autorização para participar da rede, manter o registro das transações compartilhadas (ledger) e executar o protocolo de consenso. As arquiteturas públicas permitem que tanto a visualização quanto o potencial de gerar transações possam ser realizados por todos. Já na arquitetura privada, apenas um número selecionado de nós (participantes) está autorizado a manter o registro das transações compartilhadas (O'Leary, 2017).

O Blockchain público tem como principal vantagem a falta de necessidade de uma administração central, empregando mecanismos de consenso. Porém, todos os registros devem ser guardados por todos os elos da rede, o que exige recursos computacionais adequados de cada lado da transação. Como a informação é pública, ela pode se tornar alvo de criminosos, um dos motivos pelos quais as transações contábeis e da cadeia de suprimentos, normalmente, utilizam o sistema fechado (O'Leary, 2017). Kshetri (2018) menciona que nem todas as empresas preferem um sistema público (aberto), o que permitiria que qualquer um possa participar. No caso da SCM, muitas empresas preferem o uso do sistema privado (fechado) por ser mais restrito, uma vez que o acesso só pode ser feito com a liberação de uma autoridade.

Schwab (2018) destaca que o uso do Blockchain permitirá a criação de novos modelos de manufatura e de áreas comerciais, pensamento também compartilhado por O'Leary (2017) ao enfatizar que não basta automatizar os processos para reduzir os papéis. Por outro lado, os mesmos autores também destacam os riscos envolvidos devido à ambiguidade jurídica com relação às responsabilidades no uso da tecnologia e à regulamentação do uso de dados, à exigência de nova infraestrutura de gerenciamento de riscos e governança e inexistência de padrões técnicos universais.

No ambiente organizacional, existem diferentes formas de utilizar a tecnologia com aplicações em diferentes áreas e setores, como será discutido na próxima seção.

\subsection{0 uso do Blockchain na Gestão da Cadeia de Suprimentos}

As aplicações práticas do Blockchain vão além das moedas digitais, especialmente por conta de sua auditabilidade, o que é particularmente importante quando é necessário manter a confiança entre todas as partes interessadas (Kshetri, 2018). Ko, Lee e Ryu (2018) relatam o exemplo do uso combinado de Blockchain e RFID (Radio Frequency Identification) na indústria alimentícia. Os dados de um produto referentes ao processo, armazenagem e distribuição são inseridos no RFID e instantaneamente transferidos para o sistema Blockchain via wireless. O Blockchain garante que as informações não sofram alterações e possam ser consultadas por consumidores ou órgãos governamentais por meio do link compartilhado.

Outro exemplo é a empresa Modum, conforme apresentado por Kshetri (2018), que está desenvolvendo um sistema com a Universidade de Zurich para assegurar a entrega de medicamentos. Temperatura, luz e umidade são constantemente medidas por meio de sensores durante todo o processo de transporte para garantir as condições necessárias para os fármacos. Quando o medicamento chega ao destino, os dados dos sensores são transferidos para um Blockchain e conferidos com Smart Contracts. Se as condições de transporte se mantiveram nos limites pré-estabelecidos, a carga já é liberada. Caso tenha ocorrido algum desvio, a área de recebimento é notificada para realizar avaliações.

Gao et al. (2018) comparam uma cadeia de suprimentos a um sistema distribuído que utiliza sistemas informatizados próprios, difíceis de serem integrados em uma plataforma unificada e sensível a ameaças cibernéticas. Nesse sentido, o Blockchain, com o uso do ledger descentralizado, pode representar uma possível solução para esses problemas. Kshetri (2018) expõe que a tecnologia tem potencial para auxiliar a SCM a atingir seus objetivos estratégicos como redução de custos e riscos, aumento da qualidade, velocidade, confiabilidade, sustentabilidade e flexibilidade.

Em recente pesquisa realizada, Shaw (2018) cita algumas iniciativas da utilização do Blockchain na área de Logística e SCM: o Walmart está usando Blockchain para garantir a qualidade ao rastrear 
carregamentos de carne e frutos do mar de seus fornecedores até as prateleiras de suas lojas, enquanto a Maersk's está usando a tecnologia para reduzir custos no transporte de flores da África Oriental para os países baixos. Já no comércio internacional, a tecnologia está tornando possível processar documentos e pagamentos em poucos minutos. Desse modo, ainda segundo o autor, em poucos anos, materiais e informações vão fluir entre as organizações que compõem as cadeias de suprimentos sem intervenções humanas desnecessárias.

Portanto, o Blockchain demonstra potencial para alterar a dinâmica do fluxo de informações das cadeias de suprimentos, por exemplo, pois além das transações não poderem ser alteradas por nenhuma das partes, elas podem ser verificadas por todos membros da cadeia a qualquer momento. Isso gera maior confiança entre os elos, o que é particularmente bem-vindo nos casos de cadeias que trabalham com produtos que demandam rastreabilidade, como a indústria alimentícia, farmacêutica, energia, aeronáutica, entre outras. Como os registros ao longo da cadeia são feitos de forma cumulativa e são invioláveis e disponíveis a todos, o último elo da SC (cliente final) consegue confirmar se o que está consumindo é autêntico e de qualidade (Kshetri, 2018). Em outras palavras, o Blockchain é uma tecnologia que garante a diversos stakeholders que as transações estão disponíveis, são corretas e não podem ser alteradas por nenhuma das partes sem o consenso das demais. Essa garantia não vem somente da confiança entre os interessados, mas principalmente da confiança nos algoritmos e lógicas da tecnologia, $o$ que assegura que mesmo erros humanos na manipulação de sistemas de informação não prejudicarão a qualidade dos dados disponíveis a todos.

\section{Método}

Para atingir o objetivo proposto na introdução, a metodologia selecionada para a pesquisa foi a Revisão Sistemática, devido à contemporaneidade do tema e à escassez de material acadêmico sobre Blockchain aplicado à área de SCM identificada nas pesquisas iniciais. Segundo Koller, Couto e Hohendorff (2014), a revisão sistemática surgiu do conceito de metanálise, processo de reunião e avaliação sintética de múltiplos estudos. Os autores mencionam que ao utilizar a revisão sistemática, a realização do estudo se dá em oito etapas.

A primeira etapa consiste na delimitação da questão a ser pesquisada. Assim, a pergunta formulada para esta pesquisa é: como o Blockchain pode contribuir com a Gestão da Cadeia de Suprimentos?

A segunda etapa mencionada por Koller, Couto e Hohendorff (2014) é a escolha da base de dados a ser utilizada como fonte de pesquisa. A base selecionada é o portal de periódico da Capes, por disponibilizar uma base de periódicos acadêmicos nacionais e internacionais e englobar publicações das áreas de tecnologia e SCM.

O terceiro passo da Revisão Sistemática é a seleção das palavras-chave para a busca. Em leituras iniciais sobre o tema Blockchain, foram encontrados diversos artigos sobre questões técnicas da tecnologia, como algoritmos e modelagem matemática, mas poucos estudos que pudessem orientar um gestor ou profissional que não é da área de Tecnologia da Informação sobre como a aplicação da tecnologia poderia ser utilizada na área de SCM. Buscando responder a pergunta que orienta a pesquisa, foram realizadas buscas com as palavras-chave Supply Chain Management e Blockchain, assim como com os termos em português Gestão da Cadeia de Suprimentos e Blockchain, utilizando os campos de título e o assunto dos artigos. A busca realizada, em janeiro de 2019, engloba todos os artigos publicados até dezembro de 2018, tendo como filtro adotado apenas artigos que necessariamente tenham passado pelo processo de avaliação por pares.

O quarto passo mencionado por Koller, Couto e Hohendorff (2014) é a etapa de busca e armazenamento dos resultados. Quando realizada a busca que continha as palavras-chave no título, foram encontrados onze artigos, sendo um deles repetido, o que totalizou dez artigos para avaliação. Uma segunda busca foi realizada entre os artigos que continham as duas palavras-chave no assunto, e, dessa vez, foram encontrados sete artigos, dos quais cinco se repetiam com os dados da busca anterior, totalizando doze artigos para início das análises.

A quinta etapa da pesquisa mencionada por Koller, Couto e Hohendorff (2014) é seleção dos artigos, segundo critérios de inclusão e exclusão. Como já mencionado, foi utilizado como critério de inclusão apenas artigos publicados em periódicos revisados por pares. Um dos artigos que resultaram da busca anterior é um editorial de revista e foi descartado da análise. Por se tratar de uma área recente de pesquisas, o período de publicação foi limitado a publicações efetuadas até o ano de 2018, mas não foi 
incluída uma data inicial.

A sexta, sétima e oitava etapas consistem na extração dos dados selecionados, avaliação dos artigos e sínteses e interpretação dos dados que serão apresentados nas próximas seções. As análises foram realizadas manualmente, não sendo utilizados softwares de revisão. Ao final, foram utilizados onze artigos que estão apresentados no Quadro 1.

Com o objetivo de validar as informações levantadas na revisão sistemática e verificar se elas precisavam ser ajustadas, de acordo com a recomendação de Creswell (2010), foi planejada uma verificação empírica por meio da realização de entrevistas com questões abertas envolvendo o uso do Blockchain em Gestão da Cadeia de Suprimentos e não mencionando os resultados obtidos na Revisão Sistemática. Ainda por conta da novidade do tema, não foi possível aos pesquisadores identificarem uma quantidade expressiva de entrevistados aptos a participar da investigação. Foram então selecionados, inicialmente, quatro profissionais com conhecimento nos temas do trabalho que poderiam contribuir com o processo de verificação empírica, mas apenas dois tiveram disponibilidade para tal, determinando, assim, uma amostra por conveniência.

Quadro 1: Títulos dos artigos e autores

\begin{tabular}{|l|l|c|}
\hline \multicolumn{1}{|c|}{ Nome } & \multicolumn{1}{|c|}{ Autores } & Ano \\
\hline $\begin{array}{l}\text { Will Blockchain technology revolutionize excipient supply chain } \\
\text { management? }\end{array}$ & Shireesh Apte, Nikolai Petrovsky & 2016 \\
\hline $\begin{array}{l}\text { A Novel Blockchain-Based Product Ownership management Sys- } \\
\text { tem (POMS) for Anti-Counterfeits in the Post Supply Chain }\end{array}$ & $\begin{array}{l}\text { Kentaroh Toyoda, P. Takis Mathiopoulos, } \\
\text { Iwao Sasase, Tomoaki Ohtsuki }\end{array}$ & 2017 \\
\hline $\begin{array}{l}\text { Blockchain and supply chain management: aircrafts'parts' business } \\
\text { case }\end{array}$ & Yash Madhwal \& Peter B Panfilov & 2017 \\
\hline $\begin{array}{l}\text { Configuring blockchain architectures for transaction information in } \\
\text { blockchain consortiums: The case of accounting and supply chain } \\
\text { systems }\end{array}$ & Daniel E. O'Leary & 2017 \\
\hline $\begin{array}{l}\text { Blockchain's roles in meeting key supply chain management ob- } \\
\text { jetives }\end{array}$ & Nir Kshetri & 2018 \\
\hline $\begin{array}{l}\text { CoC: A Unified Distributed Ledger Based Supply Chain Management } \\
\text { System }\end{array}$ & $\begin{array}{l}\text { Shimin Gao, Lei Xu, Xi Zhao, Yang Lu, Wei- } \\
\text { dong Shi }\end{array}$ & 2018 \\
\hline Blockchain technology for anhancing supply chain resilience & Hokey Min & 2018 \\
\hline $\begin{array}{l}\text { The impact of the blockchain on the supply chain: a theory based } \\
\text { research framework and a call for action }\end{array}$ & Horst Treiblmaier & 2018 \\
\hline $\begin{array}{l}\text { Supply Chain Management based on Blockchain: a Systematic Map- } \\
\text { ping Study }\end{array}$ & $\begin{array}{l}\text { Youness Tribis; Abdelali El Bouchti; Hous- } \\
\text { sine Bouyad }\end{array}$ & 2018 \\
\hline $\begin{array}{l}\text { Toward an ontology-driven blockchain design for supply-chain } \\
\text { provenance }\end{array}$ & Henry M. Kim e Marek Laskowski \\
\hline $\begin{array}{l}\text { A Study on the Transparent Price Tracing System in Supply Chain } \\
\text { Management Based on Blockchain }\end{array}$ & Minjae Yoo e Yoojae Won & 2018 \\
\hline
\end{tabular}

Fonte: elaborado pelos autores

A primeira entrevista foi realizada com um professor pesquisador de uma universidade pública estadual do Estado de São Paulo, com mais de dez anos de experiência em pesquisas, com atual interesse nas áreas de IoT (Internet of Things), desenvolvimento de kernel de sistema operacional para IoT, machine learning, Inteligência Atificial bioinspirada, desenvolvimento de software livre, aplicações sensíveis ao contexto e qualidade de serviço em redes de computadores. 0 professor realizou estudos sobre a possibilidade da implantação do Blockchain no campus onde trabalha.

A segunda entrevista foi realizada com um líder de serviços Blockchain, na América Latina, que trabalha em uma empresa do setor de Serviços de TI com mais de 300.000 funcionários no mercado global. Essa empresa presta serviços de Blockchain para várias outras empresas, além de representar um dos principais elos de sua cadeia de suprimentos que também utiliza a tecnologia. 0 profissional entrevistado conta com mais de quinze anos de experiência no setor financeiro, atua na integração e desenvolvimento de sistemas back-end e é especialista em Blockchain e Governança de Dados. 


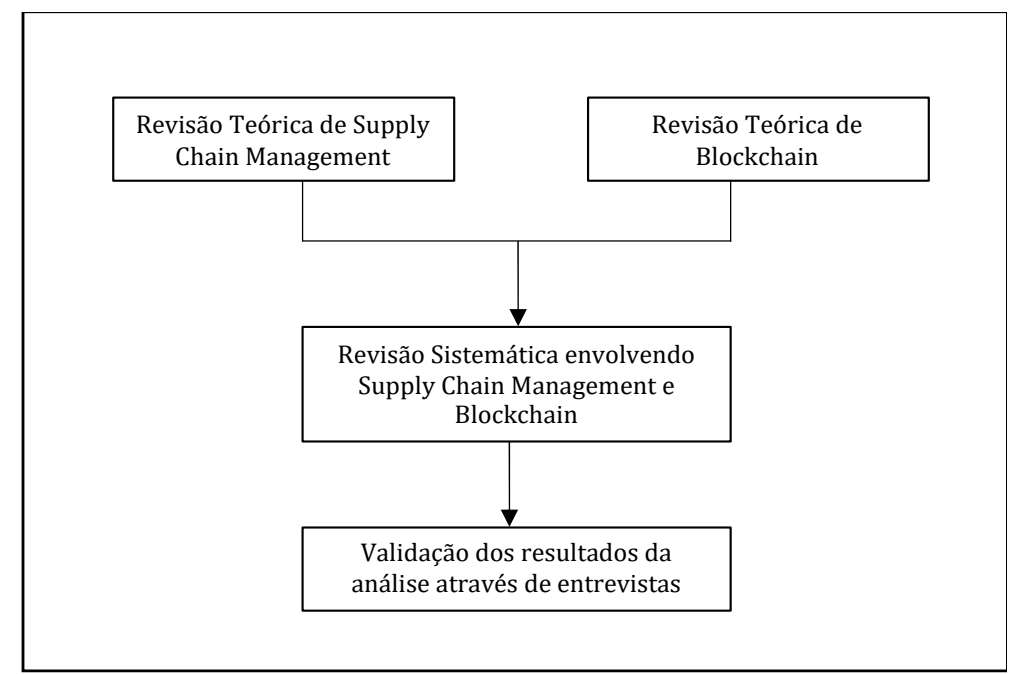

Figura 1: Metodologia da Pesquisa

Fonte: elaborado pelos autores

A metodologia utilizada para o desenvolvimento da pesquisa é apresentada na Figura 1, e os resultados do trabalho apresentados na seção 4 a seguir.

\section{O uso de Blockchain na SCM}

Entre os artigos selecionados para a análise, é possível verificar que a realização de pesquisas sobre o uso de Blockchain não tem sido apenas uma preocupação das área de tecnologias ou computacional. Os artigos sobre o uso de Blockchain em SCM estão publicados em periódicos de diferentes áreas, com predominância na área de negócios, conforme demonstra o Quadro 2.

\begin{tabular}{l} 
Quadro 2: Periódicos que publicaram sobre os temas \\
\hline Business Horizons \\
\hline DAAAM International Symposium on Inteligent Manufacturing and Automation \\
\hline IEEE Acces \\
\hline Intelligent Systems in Accounting Finance and Management \\
\hline International Journal of Information Management \\
\hline Journal of Computer Science and Technology \\
\hline Journal of Excipients and Food Chemicals \\
\hline MATEC Web of Conferences \\
\hline Intelligent Systems in Accounting, Finance and Management \\
\hline Supply Chain Management: An International Journal \\
\hline Sustainability \\
Fonte: elaborado pelos autores
\end{tabular}

Dentre os artigos que fazem parte da amostra, não houve autores que se repetiram, conforme os dados da Tabela1. Nota-se também uma diversidade de instituições que estão participando de pesquisas sobre o assunto, conforme dados do Quadro 3.

Quadro 3: Universidades envolvidas nas pesquisas sobre os temas
\begin{tabular}{|l|}
\hline Keyo University \\
\hline National and Kapodestrian University of Athens \\
\hline The University of North Carolina \\
\hline University of Houston \\
\hline Xi'na Jiaotong University \\
\hline Hassan University \\
\hline Institute for Forecasting and Futuristics \\
\hline Flinders University \\
\hline Bowling Green State University \\
\hline MODUL University Vienna \\
\hline York University \\
\hline Chungnam National University \\
\hline University of Southern California \\
\hline Fonte: elaborado pelos autores
\end{tabular}


Partindo efetivamente para a avaliação dos dados (artigos) selecionados e sua respectiva síntese e interpretação, o primeiro passo foi a análise da metodologia de pesquisa utilizada em cada artigo. Um resumo dos achados é apresentado na Figura 2. Os artigos de revisão teórica sobre os assuntos e os que apresentavam algum modelo para o uso da tecnologia foram os que mais se destacaram, com quatro artigos cada. Outros métodos foram encontrados em apenas um artigo para cada metodologia.

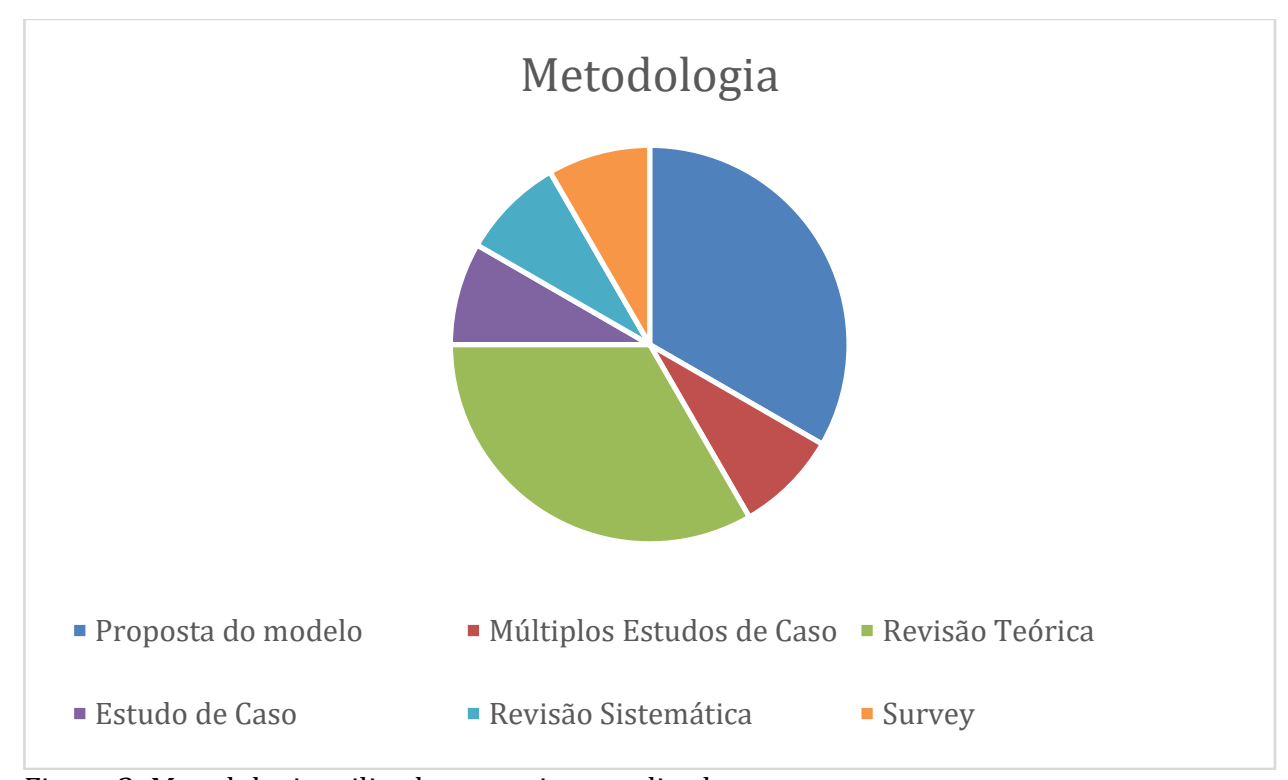

Figura 2: Metodologia utilizada nos artigos analisados

Fonte: Elaborado pelos autores

Dois artigos da amostra apresentam estudos de caso sobre a utilização do Blockchain na SCM. Um trata do estudo de um único caso e o segundo de um estudo de múltiplos casos. Os casos apresentados abrangem diferentes setores, conforme demonstra a Figura 3, destacando-se os de tecnologia, alimentício e o farmacêutico. Como o Blockchain é uma tecnologia recente e com poucos artigos publicados sobre o seu uso na SCM, os casos apresentados podem ser considerados como casos pioneiros do uso de Blockchain em SCM. Outras aplicações podem estar sendo realizadas com diferentes finalidades e setores, mas não foram apresentadas, nesta análise, por não terem sido encontrados artigos publicados na base de dados pesquisada, seguindo a metodologia adotada para a pesquisa.

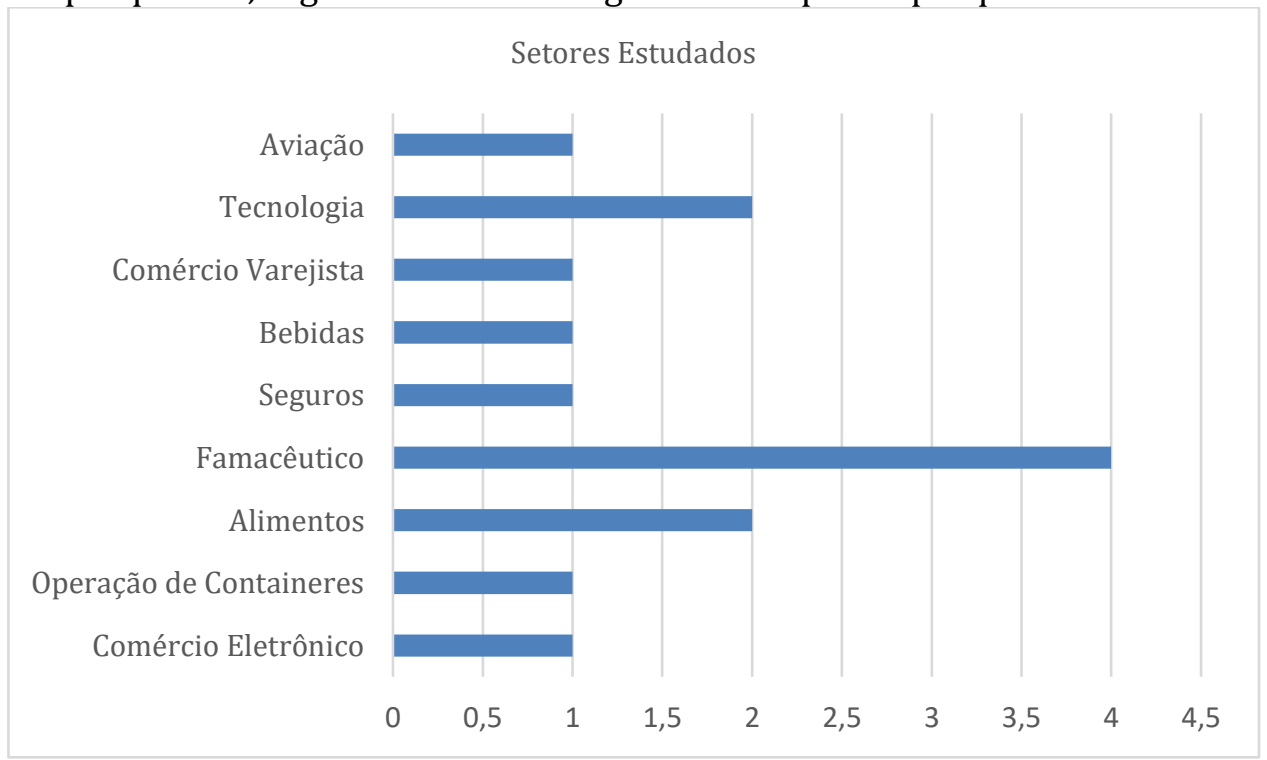

Figura 3: Setores estudados pelos artigos analisados

Fonte: Elaborado pelos autores

Conforme mencionado por Treiblmaier (2018), o Blockchain não é uma tecnologia isolada, sendo 
utilizada em conjunto com outras tecnologias. Em muitos dos artigos estudados foram citadas a utilização de outras tecnologias associadas ao Blockchain para que as cadeias de suprimentos possam atingir os objetivos esperados. Assim, o presente trabalho mapeou em quantos artigos cada tecnologia foi associada ao uso do Blockchain na aplicação em SCM. De acordo com a Figura 4, a Internet das Coisas, Big Data e RFID foram as mais citadas. Estas tecnologias permitem a integração entre o mundo digital e o físico, além de agilizarem a troca de informações em tempo real, características necessárias para o uso do Blockchain na área de SCM, principalmente, nas atividades relacionadas ao processo logístico (Min, 2018).

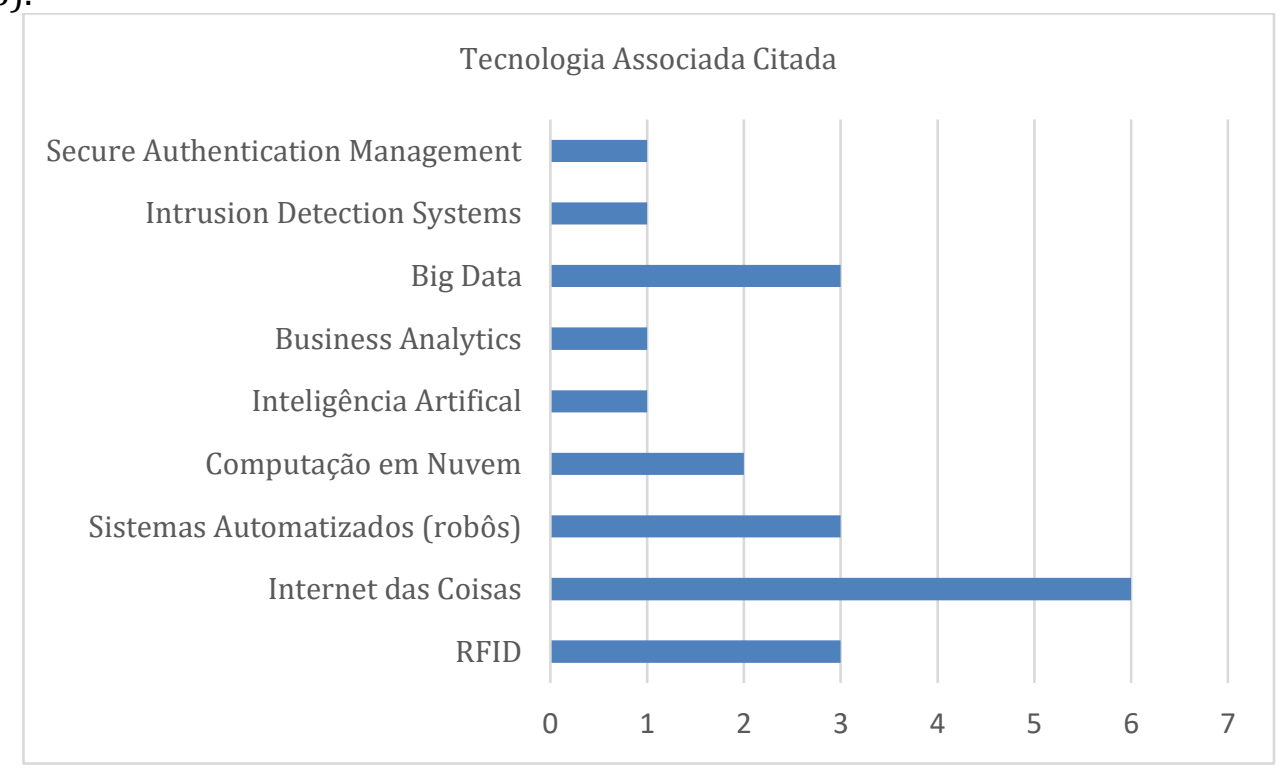

Figura 4: Tecnologias associadas citadas nos artigos analisados

Fonte: Elaborado pelos autores

Segundo Kshetri (2018), há um uso crescente de Internet das Coisas (IoT) e isto deve afetar a SCM. Quando a IoT já é utilizada para medir e rastrear os processos de SCM, os custos associados ao Blockchain acabam sendo zero ou muito baixos.

Outro exemplo é o uso de etiquetas RFID associado ao Blockchain para a verificação de produtos piratas na cadeia de suprimentos, mencionado por Toyoda, Mathiopoulos, Sasase e Ohtsuki (2017). Neste caso, um código eletrônico é atribuído a cada produto e gravado em uma etiqueta RFID. Em cada novo elo da cadeia de suprimentos, as etiquetas são verificadas e novas informações são adicionadas, garantindo, assim, a proteção contra falsificações.

Diversas características podem ser destacadas com relação ao Blockchain. Tais características são os fatores motivadores para a implantação da tecnologia tanto na área financeira quanto na SCM e outras atividades fins. Na pesquisa realizada, foram relacionadas as caraterísticas mencionadas pelos autores dos artigos selecionados a quantidade de vezes que elas aparecem nos artigos, com resultado apresentado na Figura 5. Foram explicitadas as seguintes características: segurança das informações, solidez dos dados, possibilidade de ampla visibilidade das informações, o fato de as informações serem públicas, o baixo custo para utilização, o registro único em que a informação não pode ser modificada e, também, o registro distribuído.

Conforme demonstra a Figura 5, as características mais destacadas na amostra de artigos analisada foram o registro distribuído e a imutabilidade. Gao et al. (2018) destacam a acessibilidade pública, pela qual todos os elos interessados podem ter acesso a informação; a imutabilidade, já que não é possível remover ou alterar as informações já adicionadas; e a resiliência, pois todos os participantes do sistema possuem uma cópia inteira do Blockchain. Treiblmaier (2018) também destaca como característica importante a imutabilidade, mencionando que a resistência à mudança das informações pode elevar o nível de confiança nas operações. Já Kim e Laskowski (2018) apontam que o uso de bancos de dados distribuídos e compartilhados possibilita um ambiente seguro e imutável aos dados da cadeia de suprimentos, sendo possível avaliar a proveniência dos dados mesmo sem nenhuma solicitação.

Alguns dos princípios da SCM mencionados por Bowersox et al. (2014) e Ellram e Cooper (2014), na seção 1, deste artigo, são a transparência das informações e o comportamento cooperativo, o que 
demonstra que o Blockchain, com suas características, pode ser uma importante ferramenta colaborativa para atingir tais objetivos. Isso porque, considerando que as características mais mencionadas nos artigos analisados foram o registro distribuído e a imutabilidade, a tecnologia pode permitir que todos os elos acessem os dados necessários sempre que desejarem (relação transparência com registro distribuído) e que a confiança entre as partes aumente (relação comportamento cooperativo com imutabilidade). Christopher (2011) também enfatiza a importância do gerenciamento do fluxo das informações para a realização da SCM, uma área em que o Blockchain pode contribuir muito por meio da imutabilidade, da visibilidade e segurança das informações recebidas.

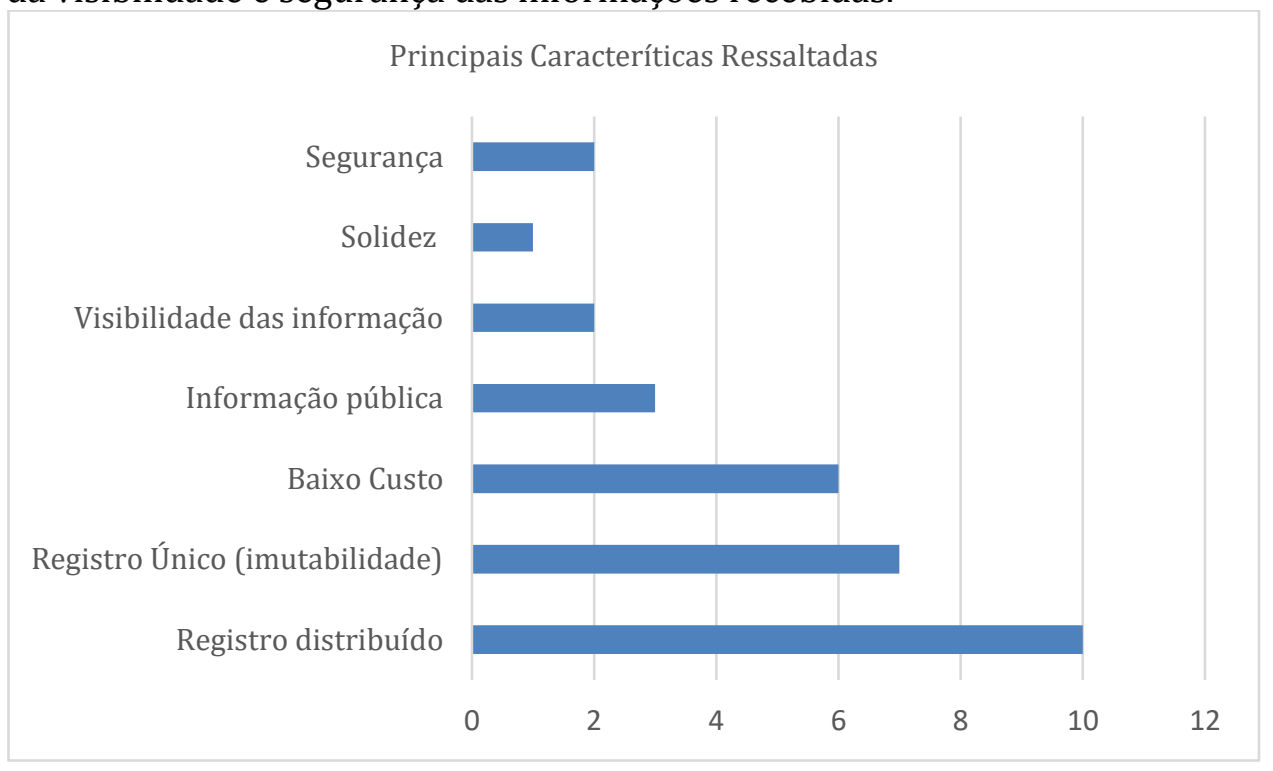

Figura 5: Características ressaltadas nos artigos analisados

Fonte: elaborado pelos autores

Outro passo da síntese e interpretação dos dados foi a verificação aprofundada sobre como o Blockchain pode colaborar com a SCM. Foram verificadas contribuições na forma como as empresas se relacionam física e digitalmente. De forma resumida, foram 21 os benefícios apresentados nos onze artigos: auxílio na redução da pirataria; certificação da origem da matéria prima; auxílio na validação de produtos e documentos, levando, assim, à eliminação de documentos; acesso aos dados que se dá em tempo real por todos os elos da cadeia de suprimentos; possibilidade de integração com robôs, sendo possível acompanhar indicadores de qualidade e produtividade por todos os elos da cadeia; possibilidade de integração com Internet das Coisas, facilitando a formação e mensuração de indicadores chave de negócios; transparência; velocidade; acessibilidade; integração entre o mundo real e o digital possível com adoção de outras tecnologias integradas; prevenção de riscos; uso de contratos inteligentes; redução de custos; o fato de não poder ser corrompido; prevenção de fraudes; segurança dos dados; manutenção do histórico das transações; possibilidade de inovação; responsabilidade e; rastreabilidade e aumento na eficiência da cadeia de suprimentos. Interessante notar como muitos destes fatores apresentados se inter-relacionam e acabam se originando nas características apresentadas na Figura 6, cujo eixo Y representa quantas vezes cada benefício foi citado ao final da análise dos onze artigos.

Dentre as contribuições do Blockchain para a SCM elencadas na Figura 6, algumas delas se destacam. Kshetri (2018) defende que o gerenciamento de identidades é a maior vantagem da tecnologia para a SCM, já que isso torna possível saber quem está realizando as ações, assim como facilita o rastreamento do progresso de remessas e entregas. Além disso, possibilita registrar as medidas de qualidade de um produto durante o processo de transporte.

Apte e Petrovsky (2016) mencionam o auxílio da tecnologia às cadeias de suprimentos com relação aos produtos ilícitos ou falsificados, pois a utilização do Blockchain permite verificar com exatidão onde o produto foi montado ou feito. Esta questão da segurança também é apresentada por Min (2018). 0 autor destaca a segurança, a redução de custos de transação e de tempo, a possibilidade de reduzir o envolvimento de terceiros, a visibilidade compartilhada em toda a cadeia de suprimentos e a possibilidade de impedir a falsificação, rastreando remessas físicas em tempo real e utilizando registros armazenados em Blockchain. 


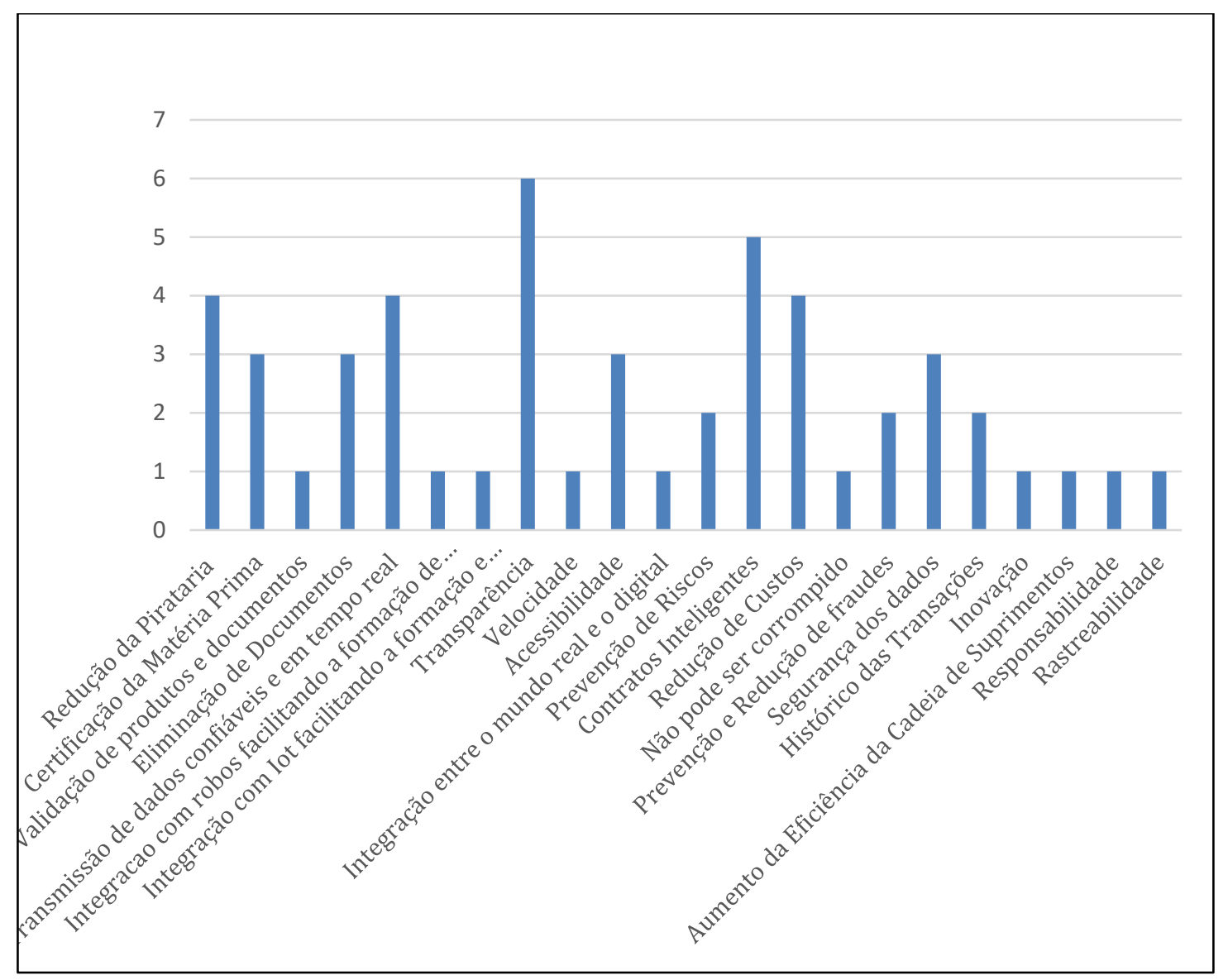

Figura 6: Contribuições do Blockchain à SCM de acordo com os artigos analisados Fonte: elaborado pelos autores

Madhwal e Panfilov (2017) salientam o valor acrescentado ao processo ao certificar que os produtos recebidos são legítimos e estão isentos de falsificação de algum componente. Esse mesmo ponto pode ser corroborado por O'Leary (2017), quando este comenta sobre a possibilidade de todos os membros da cadeia poderem visualizar as informações disponíveis no ledger. Já Tribis, Bouchti e Bouayad (2018) destacam a rastreabilidade em tempo real com informações confiáveis e alto nível de confiabilidade para todos os membros da cadeia de suprimentos, assim como o gerenciamento da informação eficiente devido à imutabilidade dos dados e à acessibilidade pública dos registros.

Yoo e Won (2018) enfatizam a maior transparência no processo de transporte, além da redução de custos com o uso dos contratos inteligentes e maior segurança da informação. Já segundo Schwab (2018), o Blockchain permite a integração do mundo físico e digital e cria identificações verdadeiramente únicas, reduzindo os custos das transações entre clientes e fornecedores.

A possibilidade de integrar o Blockchain com outras tecnologias para garantir a integridade e segurança das informações corroboram com o objetivo da SCM mencionado por Bowersox, Closs, Cooper e Bowesox (2014) de melhorar a eficiência operacional da cadeia de suprimentos e o seu posicionamento estratégico.

Todos os benefícios mencionados se alinham às necessidades da SCM citadas por Asgari et al. (2016) e Ellram e Cooper (2014) de maior agilidade e flexibilidade nas operações conjuntas. Além disso, eles podem proporcionar o atendimento ao cliente de forma eficiente e com menor custo possível, um dos maiores objetivos da SCM (Ellram \& Cooper, 2014; Pires, 2016). Outras contribuições do Blockchain para a SCM envolvem a eliminação dos trabalhos duplicados e improdutivos, aumento da velocidade, confiabilidade e flexibilidade de processos e redução dos custos mencionados por Bowersox et al. (2014).

Além dos fatores positivos relativos ao uso do Blockchain, a pesquisa também identificou aspectos negativos e desafios relacionados a esta tecnologia.

Os desafios citados por Kshetri (2018) estão relacionados à falta de regulamentações e códigos 
comerciais diferentes para cada empresa, o que se agrava em cadeias de suprimentos que atuam no cenário internacional. Além disso, as informações relacionadas a uma remessa física são imutáveis, mas não é possível evitar todas as atividades físicas e fraudulentas no mundo físico. Outro desafio mencionado pelo autor é que nem todos os países estão prontos para participar de soluções baseadas em Blockchain por conta do alto grau de informatização necessário. Membros de cadeia de suprimentos localizados em países em desenvolvimento ou menos desenvolvidos podem ter dificuldades para adotar a tecnologia em suas operações.

Tribis et al. (2018) mencionam as lacunas de regulação e barreiras jurídicas como aspectos a serem levados em consideração, assim como a ausência de padrões comuns para todas as operações. Outras barreiras citadas pelos autores e também por Min (2018) se referem à necessidade de adaptação entre as tecnologias novas e antigas e a questão da escalabilidade, uma vez que o Blockchain exige um grande poder computacional e é oferecido por poucas empresas. O'Leary (2017) identifica como barreira relacionada à implantação da tecnologia conseguir explorar a total eficiência do Blockchain. Para que isso seja possível, as organizações devem fazer uma análise e eestruturação dos processos já existentes. Segundo o autor, muitas empresas acabam simplesmente utilizando o Blockchain com o objetivo de automatizar um processo já existente ou reduzir a circulação de papéis.

Após a análise bibliográfica, foram realizadas duas entrevistas com profissionais que atuam com a tecnologia Blockchain para identificar novas contribuições e verificar as contribuições mencionadas na revisão sistemática.

\subsection{Verificação empírica}

Conforme mencionado anteriormente, o objetivo da verificação empírica foi de validar as informações levantadas na revisão sistemática e verificar se elas precisavam ser ajustadas. As entrevistas foram realizadas com questões abertas, previamente estruturadas e alinhadas à questão formulada para a revisão sistemática. Os entrevistados foram questionados, de modo geral, sobre a possibilidade da utilização do Blockchain na área de SCM e quais seriam as maiores contribuições de sua utilização.

A primeira entrevista foi realizada com um professor pesquisador. 0 entrevistado ressaltou como característica importante para o Blockchain o consenso distribuído, o qual pelo menos cinquenta por cento mais um dos participantes têm de validar que o novo bloco inserido é verdadeiro. Na opinião do pesquisador, essa tecnologia pode ser utilizada em votos, governo eletrônico, cadeias de suprimentos, entre outros. Outros exemplos incluem a utilização em transações financeiras como Bitcoin ou em registros que poderiam substituir os cartórios, o que eliminaria a necessidade e a dependência de uma instituição reguladora para validar as informações, o que ainda não é possível pelas leis vigentes.

Ainda na opinião do entrevistado, no campo da cadeia de suprimentos, o Blockchain, pode contribuir para rastrear a procedência de produtos, sua data de produção, detalhes sobre o processo de transferência, informações entre as entidades, entre outros. É possível realizar o registro de todas as movimentações desde a primeira empresa onde se iniciou o processo de transformação até o descarte do produto. Como principais contribuições do Blockchain para a Gestão da Cadeia de Suprimentos, o entrevistado citou maior confiança com relação à origem e à qualidade dos materiais, redução dos custos e eliminação de entidades de verificação e validação ou reguladoras do meio do caminho. É importante ressaltar que o pesquisador já iniciou pesquisas para o uso do Blockchain no campo universitário para a validação de documentos e informações acadêmicas que necessitam de maior confiabilidade.

Na segunda entrevista, realizada com um profissional atuante na área de Blockchain, o entrevistado ressaltou como principais contribuições da tecnologia para a SCM a eliminação de disputas entre os elos pertencentes a cadeia, o rastreamento de ativos e a automatização do ciclo de compras. Estes ganhos podem ser obtidos com a utilização dos Smart Contracts estabelecidos. 0 profissional ressaltou que os maiores ganhos estão ligados à redução das perdas e ao aumento da eficiência de toda a cadeia de suprimentos, possibilitados pela veracidade das informações e pelo rastreamento dos produtos em toda a cadeia de suprimentos.

As entrevistas apresentam resultados distintos e que se complementam, provavelmente por serem realizadas com profissionais com perfis diferentes, um mais dedicado à área de pesquisa de tecnologia e outro com perfil gerencial. Apesar disso, elas comprovam os dados levantados na revisão sistemática da literatura, além de agregar outras contribuições do Blockchain para a SCM. Na primeira entrevista, foi possível confirmar as características de segurança, a informação pública e o registro distribuído 
apresentadas na Figura 5. Dentre as formas de utilização apresentadas na Figura 6, foram citadas a certificação da matéria prima, validação de produtos e documentos, eliminação de documentos, redução de custos e rastreabilidade. A segunda entrevista não menciona diretamente as características apresentadas na Figura 5. Porém, as informações fornecidas pelo entrevistado podem ser classificadas nas características segurança e visibilidade das informações. Dentre as formas de utilização da Figura 6, foram mencionados especificamente os contratos inteligentes (Smart Contracts) e a rastreabilidade. Novas colaborações não relacionadas nos artigos foram mencionadas nesta entrevista, como a eliminação de entidades mediadoras e de disputas entre elos, a automatização de compras e a redução de perdas.

\subsection{Resultados e Discussões}

Os resultados da pesquisa demonstram diferentes formas como o Blockchain pode contribuir para a SCM, especificamente para o aumento da segurança e confiabilidade das informações, que quando são disponibilizadas de forma integrada por outras tecnologias, permitem a redução de processos de controle e de custos.

O Blockchain pode garantir maior segurança na veracidade das informações devido a sua característica de imutabilidade e registros em cadeia, o que pode ser muito utilizado no acompanhamento de todas as etapas do processo da cadeia de suprimentos, desde a matéria-prima inicial até o consumidor. Nesse sentido, essa tecnologia pode permitir a rastreabilidade do produto, garantir sua autenticidade, promover a eliminação de documentos desnecessários e eliminar fraudes em informações, considerando que todos os elos podem ter o histórico das transações feitas na cadeia. Essas possibilidades corroboram as necessidades da SCM propostas por Pires (2016) e Christopher (2011) de garantia de fluxo e segurança da informação. 0 uso combinado com outras tecnologias como o RFID, sensores e Iot, dá a tecnologia uma ampla utilização como meio de validação e certificação de produtos físicos e digitais, reduzindo custos e processos.

Outras grandes contribuições se dão pelo aumento da visibilidade das informações para todos os elos da cadeia de suprimentos por meio do código hash e pelo auxilío na automação de transações comerciais pré-estabelecidas com o uso de contratos inteligentes, colaborando para o aumento da produtividade e flexibilidade mencionados por Asgari, Nikbakhsh, Hill e Farahani (2016).

Os artigos analisados neste trabalho foram publicados em diferentes áreas do conhecimento, demonstrando a aplicabilidade da tecnologia em diferentes setores, embora grande parte das pesquisas tenham sido realizada na área farmacêutica. Porém, não é possível concluir que a tecnologia é mais utilizada por esse setor em detrimento de outros, mas sim que muitas pesquisas vêm sendo realizadas nesta área. É possível supor que como o uso da tecnologia está muito atrelado a questões de segurança e confiabilidade, ela se apresenta como um importante suporte ao gerenciamento de riscos, fator importante na cadeia de suprimentos do setor farmacêutico.

O comportamento cooperativo dos membros da cadeia de suprimentos, fator primordial para a existência da SCM, como citado por Pires (2016), Ellram e Cooper (2014) e Bowersox et al. (2014), quando combinado à segurança proporcionada pelo uso do Blockchain, pode ser uma importante fonte de redução de custos e agilidade dos processos. Esse comportamento colaborativo pode ser também um fator facilitador para a implantação da tecnologia, exigindo novos estudos sobre a sua comprovação.

A pesquisa realizada engloba publicações até dezembro de 2018 e demonstra um crescimento anual de artigos envolvendo os temas Blockchain e SCM simultaneamente. Novas publicações realizadas posteriormente podem demonstrar novos usos e estudos de casos que podem servir de exemplo para outras organizações e setores sobre como utilizar a tecnologia. Neste período, muitas publicações mencionam as questões técnicas da tecnologia e poucas demonstravam exemplos de sua aplicabilidade para servir de exemplo e base de ideias a profissionais da área de SCM que não são especialistas em tecnologia.

\section{Considerações Finais}

O Blockchain é uma tecnologia contemporânea, já conhecida no mercado financeiro, que tem se expandido para outras áreas, como a SCM, especialmente por conta dos benefícios que pode proporcionar às empresas (Kshetri, 2018).

Assim, a presente pesquisa teve como objetivo identificar como o Blockchain pode contribuir com a Gestão da Cadeia de Suprimentos. Para atingir esse propósito, ela foi realizada por meio de uma revisão 
sistemática de literatura, que identificou onze artigos para análise, e de um processo de verificação empírica conduzido por meio de duas entrevistas.

A análise dos dados indicou que a primeira publicação sobre os temas foi realizada em 2015, e novos trabalhos foram adicionados a cada ano, o que demonstra um crescimento no interesse pela área de pesquisa. Todavia, nota-se que ainda é muito pequeno o número de trabalhos conciliando o uso de Blockchain e o SCM, comprovando oportunidades de pesquisas já que, segundo O'Leary (2017) e Schwab (2018), para sua implantação serão necessárias mudanças nos processos e na forma como as empresas se relacionam. Os impactos da tecnologia e as mudanças necessárias na cadeia de suprimentos para sua implantação são importantes assuntos que carecem de mais estudos.

Os resultados da pesquisa indicam que o Blockchain é uma tecnologia que pode ser muito útil para a SCM. Para maximizar os resultados, seu uso pode estar atrelado a outras tecnologias, sendo a internet das coisas, Big Data, sistemas automatizados e RFID as mais citadas nos artigos avaliados.

As principais características do Blockchain identificadas nessa pesquisa e validadas empiricamente são: o registro distribuído, a imutabilidade e o custo, nesta ordem, o que condiz com os objetivos almejados na implantação da SCM. Segundo Bowersox et al. (2014), Christopher (2011) e Pires (2016), os objetivos da SCM estão relacionados à redução dos custos, à agregação de valor a produtos e serviços, ao aumento da lucratividade e à eficácia operacional, além da redução dos riscos em toda a cadeia.

Ao analisar as características do Blockchain, os objetivos da cadeia de suprimentos e a chance de uso conjunto com outras tecnologias, abre-se um grande número de possibilidades de aproveitamento do Blockchain na SCM e diferentes formas de colaboração que se complementam. Nos artigos analisados, foram citadas 21 diferentes formas de como esta tecnologia pode contribuir com as operações conjuntas. Exemplos validados nas entrevistas incluem maior valor dado à transparência das informações para todos os elos da cadeia, utilização dos contratos inteligentes que proporciona automação para alguns processos de troca de dados e redução de conflitos, assim como colaboração na redução da pirataria, redução de custos e transmissão de dados confiáveis e em tempo real. Outros benefícios importantes do Blockchain para a cadeia de suprimentos são a rastreabilidade, que possibilita também a certificação da origem da matéria prima, e o aumento na eficiência da cadeia como um todo. Além das vantagens identificadas na literatura, os entrevistados ainda mencionaram que a tecnologia pode proporcionar a eliminação de entidades de verificação ou reguladoras, eliminação de disputas entre os elos da cadeia, automatização do ciclo de compras e redução de perdas.

Apesar dos benefícios para a SCM, foram identificados como pontos de preocupação a necessidade de recursos computacionais robustos, o que pode prejudicar a adoção da tecnologia em países em desenvolvimento, a possibilidade de ainda existir fraudes nos produtos físicos mesmo com o uso da tecnologia e a necessidade de regulamentações, principalmente, para atuação no mercado internacional. Esses achados indicam que o Blockchain é uma tecnologia que tem muito a colaborar com a SCM, mas por ainda ser incipiente, demanda novas pesquisas para ampliar o seu campo de utilização.

Assim, entende-se que o trabalho pode contribuir com a área acadêmica especificamente ao apresentar exemplos relevantes de utilização do Blockchain na Gestão da Cadeia de Suprimentos, suprindo uma lacuna de estudos que versem sobre esses temas em âmbito nacional. Nesse sentido, o estudo pode servir de base para essas novas pesquisas de acadêmicos brasileiros. No âmbito gerencial, a pesquisa demonstrou que o Blockchain traz diversas vantagens para a SCM, mas que desafios ainda precisam ser superados. Espera-se que, analisando os achados do trabalho, gestores e administradores em geral considerem implementar a tecnologia em suas cadeias de suprimentos, levando em conta os benefícios detalhados e as barreiras a serem superadas.

\section{Referências}

Apte, S., \& Petrovsky, N. (2016). Will blockchain technology revolutionize excipient supply chain management? Journal of Excipients and Food Chemicals, 7(3), 76-78.

Asgari, N., Nikbakhsh, E., Hill, A., \& Farahani R. Z. (2016). Supply chain management 1982-2015: A review. IMA Journal of Management Mathematics, 27(3), 353-379.

Bowersox, D., Closs, D., Cooper, M., \& Bowersox, J. C. (2014). Gestão Logística da Cadeia de Suprimentos. Porto Alegre: AMGH.

Christopher, M. (2011). Logística e Gerenciamento da Cadeia de Suprimentos: criando redes que agregam valor. São Paulo: Cengage Learning. 
Creswell, J. W. (2010). Projeto de Pesquisa: métodos qualitativo, quantitativo e misto. Porto Alegre: Artmed.

Ellram, L.M., \& Cooper, M. (2014). Supply Chain Management: It's all about yhe journey, not the destination. SCM: Journey, 50(1), 8-20.

Gao, Z., Xu, L., Chen L., Zhao, X., Lu, Y., \& Shi, W. (2018). CoC: A Unified Distributed Ledger Based Supply Chain Management System. Journal of Computer Science and Technology, 33(2), 237-248.

Kim, H. M. \& Laskowski, M. (2018). Toward an ontology-driven blockchain design for supply-chain provenance. Intelligent Systems in Accounting, Finance and Management, 25(1), 18-27.

Ko, T., Lee, J., \& Ryu, D. (2018). Blockchain Technology and Manufacturing Industry: Real-Time Transparency and Cost Savings. Sustainability, 10, 1-20.

Koller, S. H., Couto, M. C. P., \& Hohendorff, J. V. (2014). Manual de Produção Científica. Porto Alegre: Penso.

Kshetri, N. (2018). 1 Blockchain's roles in meeting key supply chain management objectives. International Journal of Information Management, 39, 80-89.

Madhwal, Y., \& Panfilov, P. (2017). Blockchain And Supply Chain Management: Aircrafts' Parts' Business Case. $28^{\text {th }}$ DAAAM International Symposium on Intelligent Manufacturing and Automation, Vienna, Austria, 1051-1056.

Min, H. (2018). Blockchain technology for enhancing supply chain resilience. Business Horizons, 62(1), $35-45$.

O'Leary, D. E. (2017). Configuring blockchain architectures for transaction information in blockchain consortiums: The case of accounting and supply chain systems. Intelligent Systems in Accounting, Finance and Management, 24(4), 138-147.

Pires, S.R.I. (2016). Gestão da Cadeia de Suprimentos (Supply Chain management): conceitos, estratégias, práticas e casos. São Paulo: Atlas.

Queiroz, M. M., \& Wamba, S.F. (2019). Blockchain adoption challenges in supply chain: An empirical investigation of the main drivers in India and the USA. International Journal of Information Management, 46, 70-82.

Schwab, K. (2018). Aplicando a quarta revolução industrial. São Paulo: Edipro.

Shaw, J. (2018). The Future of Blockchain in Supply Chain. Apics Magazine, 28, 46-50. Recuperado em 10 setembro, 2018 de https://www.apics.org/mediaarchive/apicsmagazine/2018/Q32018APICSMAG.pdf.

Toyoda, K., Mathiopoulos, P.T., Sasase, I., \& Ohtsuki, T. (2017). A Novel Blockchain-Based Product Ownership Management System (POMS) for Anti-Counterfeits in the Post Supply Chain. IEEE Access, 5, 17465-17477.

Treiblmaier, H. (2018). The impact of the blockchain on the supply chain: a theory-based research framework and a call for action. Supply Chain Management, 23(6), 545-559.

Tribis, Y., Bouchti, A. El, \& Bouayad, H. (2018). Supply Chain Management based on Blockchain : A Systematic Mapping Study. In MATEC Web of Conferences, 200, 0020.

Yoo, M., \& Won, Y. (2018). A study on the transparent price tracing system in supply chain management based on blockchain. Sustainability (Switzerland), 10(11). 